\title{
Hedged Monte-Carlo: low variance derivative pricing with objective probabilities
}

\author{
Marc Potters ${ }^{\dagger}$, Jean-Philippe Bouchaud ${ }^{\dagger, *}$ and Dragan Sestovic ${ }^{\dagger}$ \\ $\dagger$ Science \& Finance, Capital Fund Management, \\ 109-111 rue Victor Hugo, 92532 Levallois CEDEX, FRANCE \\ http://www.science-finance.fr \\ * Service de Physique de l'État Condensé, Centre d'études de Saclay, \\ Orme des Merisiers, 91191 Gif-sur-Yvette CEDEX, FRANCE
}

August 9, 2000

\begin{abstract}
We propose a new 'hedged' Monte-Carlo (HMC) method to price financial derivatives, which allows to determine simultaneously the optimal hedge. The inclusion of the optimal hedging strategy allows one to reduce the financial risk associated with option trading, and for the very same reason reduces considerably the variance of our HMC scheme as compared to previous methods. The explicit accounting of the hedging cost naturally converts the objective probability into the 'risk-neutral' one. This allows a consistent use of purely historical time series to price derivatives and obtain their residual risk. The method can be used to price a large class of exotic options, including those with path dependent and early exercise features.
\end{abstract}

\section{Introduction}

The Black and Scholes model for options has two truly remarkable properties: (i) one can find a 'perfect' hedging strategy that eliminates risk entirely, and (ii) the option price does not depend at all on the average return of the underlying asset [1, 2, 3]. This last property shows that the option price is not simply the (discounted) average of the future pay-off over the objective (or historical) probability distribution, as one would have naively expected. This is even more striking in the case of the Cox-Ross-Rubinstein binomial model [4, 2] where the pricing measure is completely unrelated to the actual distribution of returns. These two models have deeply influenced financial mathematics, and have lead to the development 
of a rather abstract and general framework for derivative pricing. Within this framework, the fundamental result is that the requirement of absence of arbitrage opportunities is equivalent to the existence of a 'risk-neutral probability measure' (a priori distinct from the objective one), such that the price of a derivative is indeed its (discounted) average pay-off, but where the average is performed over the risk-neutral distribution rather than over the objective distribution [5, 6]. It is thus a common belief that the knowledge of the 'true' probability distribution of returns is a useless information to price options. The credence is rather that the relevant risk neutral distribution is somehow 'guessed' by the market. A possibility is to reconstruct this risk neutral distribution from quoted option prices, and to use this information to price other instruments consistently (for example exotic options) [2, 7]. In many cases, however, exact analytical expressions for these exotic options are not available and numerical methods must be used. A very versatile method, which allows to price complicated path dependent options, is the Monte-Carlo method, where paths are generated with a weight consistent with the risk-neutral distribution. Efficient numerical techniques have been developed, in particular in [8], to implement this 'risk neutral Monte-Carlo' (RNMC).

However, in most models of stock fluctuations, except for very special cases, risk in option trading cannot be eliminated, and strict arbitrage opportunities do not exist, whatever the price of the option. That risk cannot be eliminated is furthermore the fundamental reason for the very existence of option markets. It would thus be more satisfactory to have a theory of options where the objective stochastic process followed by the underlying asset was used to compute the option price, the hedging strategy, and the residual risk. The latter is clearly important to estimate for risk control purposes. A natural framework for this is the risk minimization approach developed by several authors [9, 10, 11, 12, 13], where the optimal trading strategy is determined such that the chosen measure of risk is minimized (for example the variance of the wealth balance, although other choices are possible [12, 14]). The 'theoretical' price is then obtained using a fair game argument. Interestingly, this framework allows one to recover exactly the Black-Scholes results when the objective probabilities are log-normal, and when the continuous time limit is taken (this is shown in details in [12]). In particular, the average trend indeed completely disappears from the price and hedge.

The aim of this paper is to present a very general Monte-Carlo scheme based on this approach, that we call 'hedged Monte-Carlo' (HMC). The method, which has been inspired in part by the least square method (LSM) of Longstaff and Schwartz [8], shares with it the property that it can price a wide variety of exotic options including those with path-dependent or early exercise features. On top of that, the HMC has at least four major advantages over RNMC:

- The most important one is considerable variance reduction. This is related to the fact that the financial risk arising from the imperfect replication of the option by the hedging strategy is directly related to the variance 
of the Monte-Carlo simulation. When minimizing the former by choosing the optimal strategy, we automatically reduce the latter. The standard deviation of our results are typically five to ten times smaller than with RNMC, which means that for the same level of precision, the number of trajectories needed in the Monte-Carlo is up to a hundred times smaller.

- The HMC method provides not only a numerical estimate of the price of the derivative, but also of the optimal hedge (which may be different from the Black-Scholes $\Delta$-hedge for non Gaussian statistics) and of the residual risk.

- The method does not rely on the notion of risk neutral measure and can be used with any model of the true dynamics of the underlying (even very complex), in particular those for which the risk neutral measure is unknown and/or not uniquely defined.

- Last, but not least, the HMC method allows one to use purely historical data to price derivatives, short-circuiting the modeling of the underlying asset fluctuations. These fluctuations are known to be of a rather complex statistical nature, with fat-tailed distributions, long-range volatility correlations, negative return-volatility correlations, etc. [15, 16, 12, 17]. Within the HMC method, one can directly use the historical time series of the asset to generate the paths. The fact that a rather small number of paths is needed to reach good accuracy means that the length of the historical time series need not be very large.

\section{Basic principles of the method}

Option pricing always requires to work backwards in time. This is because the option price is exactly known at maturity, where it is equal to the pay-off. As with other schemes, we determine the option price by working step by step for maturity $t=N \tau$ to the present time $t=0$, the unit of time $\tau$ being, for example, one day. The price of the underlying asset at time $k \tau$ is denoted as $x_{k}$ and the price of the derivative is $\mathcal{C}_{k}$. We assume for simplicity that $\mathcal{C}_{k}$ only depends on $x_{k}$ (and of course on $k$ ). However, the method can be generalized to account for a dependence of $\mathcal{C}_{k}$ on the volatility, interest rate, etc., or to price multi-dimensional options (such as interest rate derivatives). We therefore also introduce the hedge $\phi_{k}\left(x_{k}\right)$, which is the number of underlying asset in the portfolio at time $k$ when the price is equal to $x_{k}$. Within a quadratic measure of risk, the price and the hedging strategy at time $k$ is such that the variance of the wealth change $\delta W_{k}$ between $k$ and $k+1$ is minimized. More precisely, we define the local 'risk' $\mathcal{R}_{k}$ as:

$$
\mathcal{R}_{k}=\left\langle\left(\mathcal{C}_{k+1}\left(x_{k+1}\right)-\mathcal{C}_{k}\left(x_{k}\right)+\phi_{k}\left(x_{k}\right)\left[x_{k}-x_{k+1}\right]\right)^{2}\right\rangle_{o},
$$


where $\langle\ldots\rangle_{o}$ means that we average over the objective probability measure (and not the risk-neutral one!). As shown in [12], the functional minimization of $\mathcal{R}_{k}$ with respect to both $\mathcal{C}_{k}\left(x_{k}\right)$ and $\phi_{k}\left(x_{k}\right)$ gives equations that allow one determine the price and hedge, provided $\mathcal{C}_{k+1}$ is known. Note that we have not included interest rate effects in Eq. (11). When the interest rate $r$ is non zero, one should consider the following modified equation:

$$
\mathcal{R}_{k}=\left\langle\left(e^{-\rho} \mathcal{C}_{k+1}\left(x_{k+1}\right)-\mathcal{C}_{k}\left(x_{k}\right)+\phi_{k}\left(x_{k}\right)\left[x_{k}-e^{-\rho} x_{k+1}\right]\right)^{2}\right\rangle_{o},
$$

where $\rho=r \tau$ is the interest rate over an elementary time step $\tau$.

In order to implement this numerically, we parallel the LSM method of Longstaff and Schwartz, developed within a risk-neutral approach [8]. We generate a set of $N_{\mathrm{MC}}$ Monte-Carlo trajectories $x_{k}^{\ell}$, where $k$ is the time index and $\ell$ the trajectory index. We decompose the functions $\mathcal{C}_{k}$ and $\phi_{k}$ over a set of $M$ appropriate basis functions $C_{a}(x)$ and $F_{a}(x)$ :円

$$
\mathcal{C}_{k}(x)=\sum_{a=1}^{M} \gamma_{a}^{k} C_{a}(x) \quad \phi_{k}(x)=\sum_{a=1}^{M} \varphi_{a}^{k} F_{a}(x) .
$$

In other words, we solve the minimization problem with the variational space spanned by the functions $C_{a}(x)$ and $F_{a}(x)$. This leads to a major simplification since now we have a linear optimization problem in terms of the coefficients $\gamma_{a}^{k}, \varphi_{a}^{k}$, for which efficient numerical algorithms are available [18]. These coefficients must be such that:

$$
\sum_{\ell=1}^{N_{\mathrm{MC}}}\left(e^{-\rho} \mathcal{C}_{k+1}\left(x_{k+1}^{\ell}\right)-\sum_{a=1}^{M} \gamma_{a}^{k} C_{a}\left(x_{k}^{\ell}\right)+\sum_{a=1}^{M} \varphi_{a}^{k} F_{a}\left(x_{k}^{\ell}\right)\left[x_{k}^{\ell}-e^{-\rho} x_{k+1}^{\ell}\right]\right)^{2}
$$

is minimized. Those $N$ minimization problems (one for each $k=0, \ldots, N-1$ ) are solved working backwards in time with $\mathcal{C}_{N}(x)$ the known final pay-off function.

Although in general the optimal strategy is not equal to the Black-Scholes $\Delta$ hedge, the difference between the two is often small, and only leads to a second order increase of the risk [12]. Therefore, one can choose to work within a smaller variational space and impose that:

$$
\varphi_{a}^{k} \equiv \gamma_{a}^{k} \quad F_{a}(x) \equiv \frac{d C_{a}(x)}{d x}
$$

This will lead to exact results only for Gaussian processes, but reduces the computation cost by a factor two.

\footnotetext{
${ }^{1}$ For numerical purposes, these basis functions have been chosen to be piecewise linear for $F_{a}$ and piecewise quadratic for $C_{a}$, with adaptive breakpoints.
} 


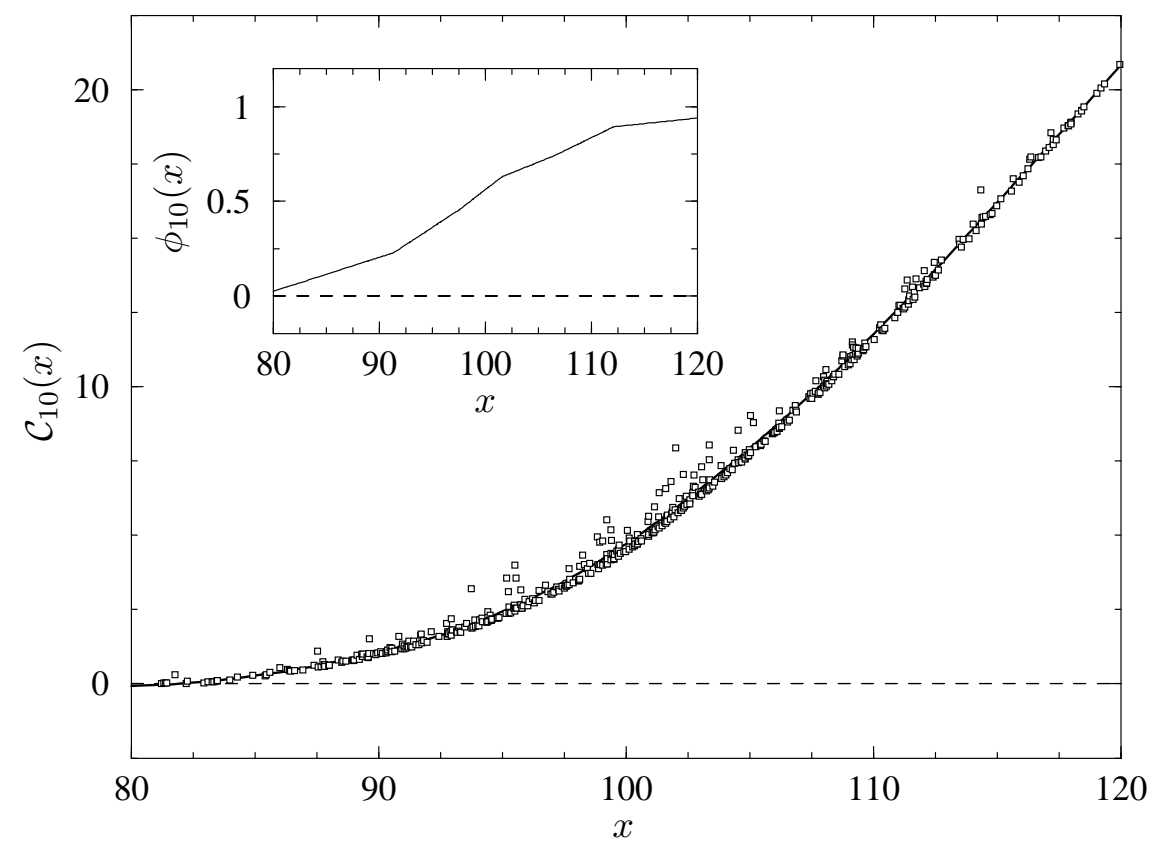

Figure 1: Option price as a function of underlying price for a HMC simulation. The full line corresponds to the fitted option price $\mathcal{C}_{10}(x)$ at the tenth hedging step $(k=10)$ of a simulation of length $N=20$. Square symbols correspond to the option price on the next step, corrected by the hedge, Eq. (6) for individual Monte-Carlo trajectories. Inset: Hedge as a function of underlying price at the tenth step of the same simulation.

\section{$3 \quad$ Numerical results for the Black-Scholes model}

We have first checked our HMC scheme when the paths are realizations of a (discretized) geometric random walk. We have priced an at the money three month European option, on an asset with $30 \%$ annualized volatility and a drift equal to the risk-free rate which we set to $5 \%$ per annum. The number of time intervals $N$ is chosen to be 20 . The initial stock and strike price are $x_{0}=x_{s}=100$, and the corresponding Black-Scholes price is $\mathcal{C}_{0}^{B S}=6.58$. The number of basis functions is $M=8$. We run 500 simulations containing 500 paths each, for which we extract the average price and standard deviation on the price. An example of the result of linear regression is plotted in Fig. 1. Each data point corresponds to one trajectory of the Monte-Carlo at one instant of time $k$, and represents the quantity:

$$
e^{-\rho} \mathcal{C}_{k+1}\left(x_{k+1}\right)+\phi_{k}\left(x_{k}\right)\left[x_{k}-e^{-\rho} x_{k+1}\right],
$$

as a function of $x_{k}$. The full line represents the result of the least-squared fit, form which we obtain $\mathcal{C}_{k}\left(x_{k}\right)$. We show in the inset the corresponding hedge $\phi_{k}$, that was constrained in this case to be the $\Delta$ hedge. 


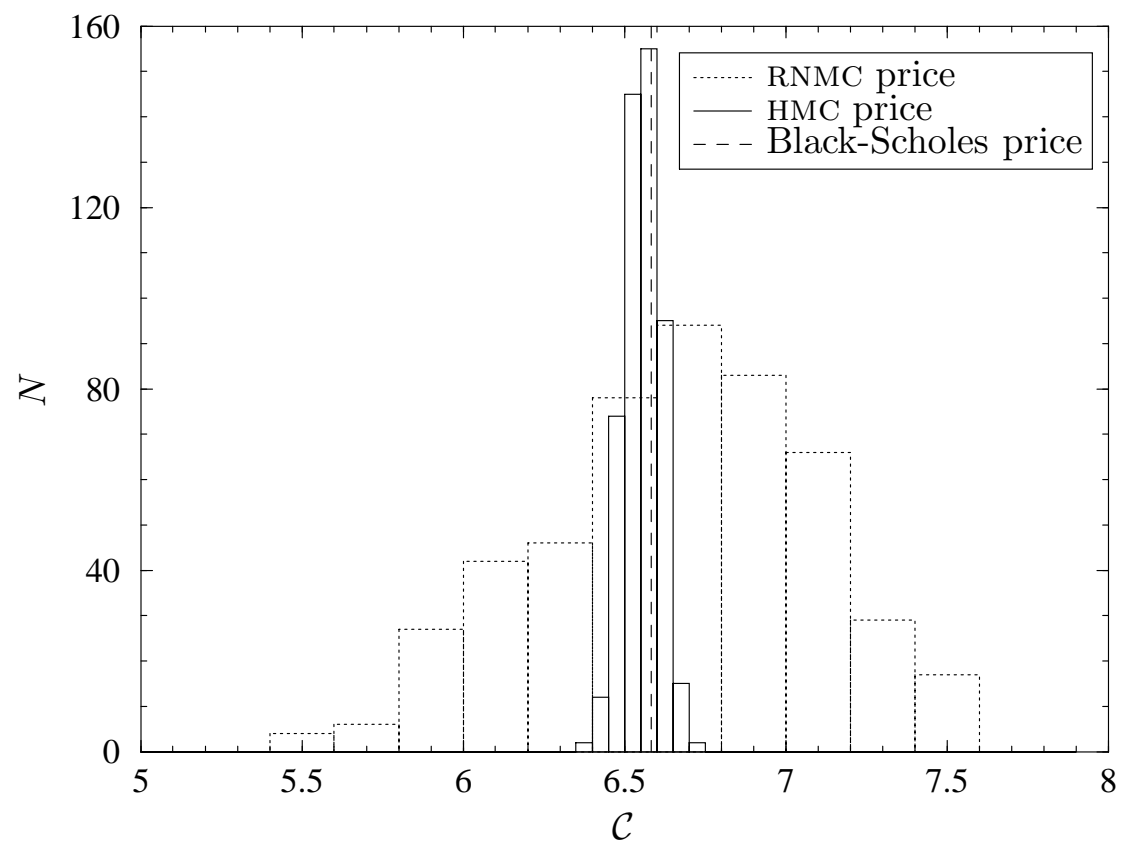

Figure 2: Histogram of the option price as obtained of $500 \mathrm{MC}$ simulations with different seeds. The dotted histogram corresponds to the RNMC and the full histogram to the HMC. The dotted line indicates the exact Black-Scholes price. Note that on average both methods give the correct price, but that the HMC has an error that is more than seven time smaller than that of the RNMC.

We obtain the following numerical results. For the RNMC (un-hedged) scheme, we obtain $\mathcal{C}_{0}^{R N}=6.68$ with a standard deviation of 0.44 . For the HMC, we obtain $\mathcal{C}_{0}^{H}=6.55$ with a standard deviation of 0.06 , seven times smaller than with the RNMC. This variance reduction is illustrated in Fig. 2, where we show the histogram of the MC results both for the un-hedged case (full bars) and for the hedged case (dotted bars).

Now we set the drift to $30 \%$ annual. The Black-Scholes price, obviously, is unchanged. A naive un-hedged Monte-Carlo scheme with the objective probabilities would give a completely wrong price of $10.72,60 \%$ higher than the correct price, with a standard deviation of 0.56 . On the other hand, the HMC indeed produces the correct price (6.52) with a standard deviation of 0.06 . The RNMC scheme in this case simply amounts to setting 'by hand' the drift to risk-free rate, and therefore obviously gives back the above figures.

Therefore, we have checked that in the case of a geometric random walk, the HMC indeed gets rid of the drift and reproduces the usual Black-Scholes results, as it should. This allows us to confidently extend the method to other type of options and other random processes. 


\section{American and other exotic options}

The HMC method can be used to reduce the Monte Carlo error for all types of exotic options. We illustrate this point by showing how the method can be extended to price an American put option. In order to implement the early exercise condition, one can simply replace $\mathcal{C}_{k+1}\left(x_{k+1}\right)$ in Eq. (4) by $\max \left(\mathcal{C}_{k+1}\left(x_{k+1}\right), x_{s}-x_{k+1}\right)$, where $x_{s}$ is the strike price. We have chosen a slightly different implementation, where we first find the early exercise point $x_{k+1}^{*}$ and exercise all options for which $x_{k+1}<x_{k+1}^{*}$.

We have tested the method on a one year American put option on a stock following a standard log-Brownian process. We follow the choice of parameters made in [8] in order to compare our results with theirs. The initial price and the strike are set to $x_{0}=x_{s}=40$, the volatility to $20 \%$ annual and the risk-free rate and the drift to $6 \%$. As a benchmark price we use the value 2.314 (quoted in [8]) computed using a very accurate finite difference method. We computed the price within the HMC using $N_{\mathrm{MC}}=500$ paths and $M=8$ basis functions. To measure the accuracy of the method, we ran the Monte Carlo 500 times with different random seeds. The average price found was 2.302 with a standard deviation (around the true value 2.314) of 0.032 .

We also used the least-square method (LSM) of [8] with the same parameters ( $N_{\mathrm{MC}}=500$ paths and $M=8$ basis functions). Note that the LSM used in [8] differs from the HMC in the following way: (i) the hedge is not used in the leastsquare fit Eq. (田), (ii) on subsequent iterations the option price is kept as the discounted pay-off on that particular path (final pay-off or early exercise value) and (iii) the final option price is given as the average pay-off of all paths. In this framework the least-square fit only serves to find the early exercise point. Therefore, for European options, the LSM is identical to the standard RNMC.

The average price within the LSM is found to be 2.423 with a standard deviation around the true value of 0.170 , five times larger than for the above quoted 0.032 for the HMC. These numbers are compatible with those found in [8] where the error quoted is 0.01 , i.e. 17 times smaller but with 200 times more paths and 2.5 times more intermediate points.

Obviously, the same variance reduction would hold for other exotic paths, as those discussed in [8]. We have not investigated in depth the optimal values to be given to the parameters $M$ and $N_{\mathrm{MC}}$, or the choice of the basis functions that minimize the computation cost for a given accuracy. These are implementation issues that are beyond the scope of the present paper.

\section{Purely historical option pricing}

We now turn to the idea of a purely historical HMC pricing scheme. We price a one month (21 business days) option on Microsoft Corp., hedged daily, with zero 


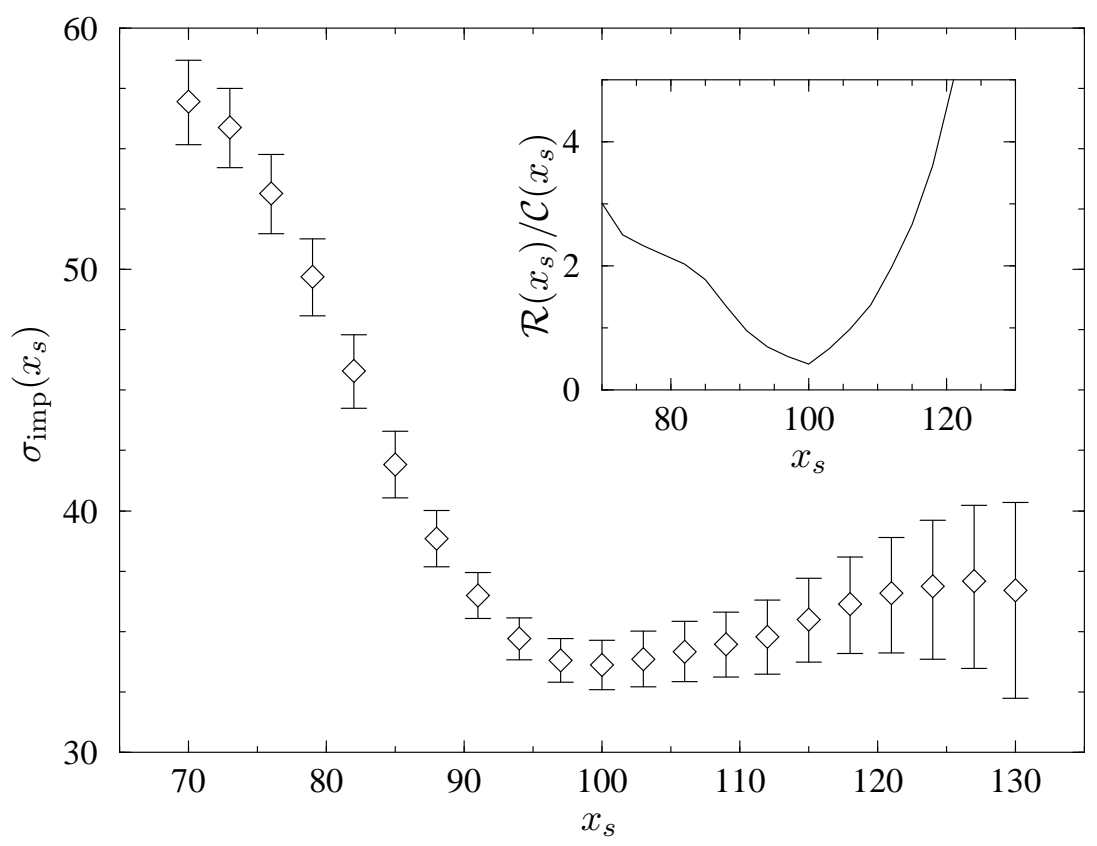

Figure 3: Smile curve for a purely historical HMC of a one-month option on Microsoft (volatility as a function of strike price). The error bars are estimated from the residual Monte-Carlo error. The inset shows the residual risk as a function of strike normalized by the 'time-value' of the option (i.e. by the call or put price, whichever is out-of-the-money).

interest rates. We used 2000 paths of length 21 days, obtained form the time series of Microsoft during the period May 1992 to May 2000. The initial price is always renormalized to 100. We use a set of $M=10$ basis functions, and keep with the simple $\Delta$-hedge. From our numerically determined option prices, we extract an implied Black-Scholes volatility by inverting the Black-Scholes formula and plot it as a function of the strike, in order to construct an implied volatility smile. The result is shown in Fig. 3, together with the error bars, obtained from the residual risk of the hedged options't]. The residual risk itself, divided by the call or the put option price (respectively for out of the money and in the money call options), is given in the inset. We find that the residual risk is around $42 \%$ of the option premium at the money, and rapidly reaches $100 \%$ when one goes out of the money. These risk numbers are comparable to those obtained on other options of similar maturity (see [12]), and are much larger than the residual risk that one would get from discrete time hedging effects in a Black-Scholes world.

The smile that we obtain has a shape quite typical of those observed on option

\footnotetext{
${ }^{2}$ The error on the price is given by the residual risk divided by the square-root of the number of independant paths. Since we over-sample the paths, we use $2000 \times 2 / 21 \approx 190$ as the effective number of paths.
} 
markets. However, it should be emphasized that we have neglected the possible dependence of the option price on the local value of the volatility. This could be taken into account by letting the function $\mathcal{C}_{k}$ depend not only on $x_{k}$ but also on the value of some filtered past volatility $\sigma_{k}$.

\section{Conclusion and prospects}

We have presented what we believe to be a very useful Monte-Carlo scheme, which closely follows the actual history of a trader hedged portfolio. The inclusion of the optimal hedging strategy allows one to reduce the financial risk associated to option trading, and for the very same reason the variance of our HMC scheme as compared to the previously discussed RNMC schemes. The explicit accounting of the hedging cost naturally converts the objective probability into the 'riskneutral' one. This allows a consistent use of purely historical time series to price derivatives and obtain their residual risk. We believe that there are many extensions and applications of the scheme, for example to price interest rate derivatives with faithful historical models (such as the one proposed in [19]), and market hedging instruments. With some modifications and extra numerical cost, the method presented here could be used to deal with transaction costs, or with non quadratic risk measures (VaR hedging).

\section{Acknowledgments}

We thank J.-P. Aguilar, A. Matacz and M. Bazant for interesting discussions.

\section{References}

[1] F. Black, M. Scholes, Journal of Political Economy, 81637 (1973).

[2] J.C. Hull Futures, Options and Other Derivative Securities, Prentice Hall (1997).

[3] P. Wilmott, J.N. Dewynne, S.D. Howison, Option pricing, Mathematical methods and Computation, Oxford University Press (1993).

[4] J. Cox, S. Ross, M. Rubinstein, Journal of Finance Economics, 7229 (1979).

[5] M. Harrison, S. Pliska, Stochastic processes and their applications, 11215 (1981).

[6] M. Baxter, A. Rennie, Financial Calculus, Cambridge University Press (1997). 
[7] see e.g. B. Dupire in Mathematics of Derivative Securities, M. Dempster, S. Pliska Edts, Cambridge University Press (1997).

[8] F. Longstaff, E. Schwartz, "Valuing American Options by Simulation: A Simple least square techniques," UCLA working paper 25-98, June 1999.

[9] M. Schweizer, The Annals of Probability, 22, 1536 (1994).

[10] M. Schäl, Mathematics of Operations Research, 19, 121 (1994).

[11] J.-P. Bouchaud, D. Sornette, J. Phys. I France, 4, 863 (1994).

[12] J.-P. Bouchaud, M. Potters, Theory of Financial Risks (in French), AléaSaclay, Eyrolles, Paris (1997); idem (in English), Cambridge University Press (2000).

[13] E. Aurell, S. Simdyankin, Int. J. of Theo. Appl. Finance, 1, 1 (1998).

[14] F. Selmi, J.-P. Bouchaud, "Hedging large risks reduces transaction costs", e-print cond-mat/0005148, submitted to RISK Magazine; B. Pochart, J.-P. Bouchaud, work in progress.

[15] for a review, see, e.g. D.M. Guillaume, M.M. Dacorogna, R.D. Davé, U.A. Müller, R.B. Olsen, O.V. Pictet, Finance and Stochastics, 195 (1997).

[16] R. Mantegna, H.E. Stanley, An introduction to Econophysics, Cambridge University Press (1999); V. Plerou, P. Gopikrishnan, L.A. Amaral, M. Meyer, H.E. Stanley, Phys. Rev., E60 6519 (1999).

[17] J.-F. Muzy, J. Delour, E. Bacry, "Modelling fluctuations of financial time series: from cascade process to stochastic volatility model," e-print condmat/0005400.

[18] W.H. Press, B.P. Flannery, S.A. Teukolsky, W.T. Vetterling, Numerical Recipes. The art of scientific computing, Cambridge University Press (1986).

[19] J.-P. Bouchaud, N. Sagna, R. Cont, N. ElKaroui, M. Potters, Applied Mathematical Finance, 6209 (1999); A. Matacz, J.-P. Bouchaud, "An empirical investigation of the Forward Interest Rate term structure," e-print condmat/9907297, to be published in Int. J. of Theo. Appl. Finance. 